\title{
Impact of Practice Effect on Each Difficulty of Cutting Skill
}

\author{
Takafumi Higashi \\ Japan Advanced Institute of \\ Science and Technology \\ Nomi, Ishikawa 9231292, Japan Nomi, Ishikawa 9231292, Japan \\ htakafumi@acm.org \\ Hideaki Kanai \\ Japan Advanced Institute of \\ Science and Technology \\ hideaki@acm.org
}

\begin{abstract}
In this paper, we aim to measure the cutting skill for creative paper cutting and increase practice effect. The practice effect changes according to the difficulty level of the cutting pattern and the skill level of the user. The cutting pattern of the picture consists of a straight line and a curved line, and we generalized the index of difficulty $(I D)$ based on Steering law. One of cutting skills that shows the difference between novices and experts is time to cut. Besides, we developed a system consisting of a drawing display and a stylus with a knife to measured the cutting movement times (MT). The system measures $M T$ according to the $I D$ of the cutting pattern. We confirmed skill improvements by measuring changes in $M T$ with various patterns. Additionally, we measured the reduction rate of $M T$ to investigate the effectiveness of practice with various IDs. As a result, we confirmed the difference in practice efficacy according to each IDs.
\end{abstract}

\section{Author Keywords}

Paper-cutting task; Steering law; Human motor performance; Stylus

\section{ACM Classification Keywords}

H.5.m [Information interfaces and presentation (e.g., $\mathrm{HCl}$ )]: classroom to make digital or hard copies of all or part of this work for personal or for profit use is granted without fee provided that copies are not made or distributed on the first page. Copyrights for components of this work owned by others than $A C M$ must be honored. Abstracting with credit is permitted. To copy otherwise, or republish, to post on servers or to redistribute to lists, requires prior specific permission and/or 29-October 3, 2018, Oslo, Norway @2018 Association for Computing Machinery. ACM ISBN $978-1-4503-6437-9 / 18 / 09 \ldots \$ 15.00$

DOI:https://doi.org/10.1145/3240167.3240236 


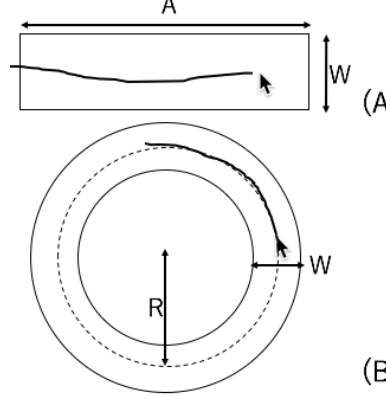

(B)

Figure 1: For a straight tunnel, $A$ is the length, and $\mathrm{W}$ its width $(\mathrm{A})$. For a circular tunnel, the movement amplitude $A$ is equal to the circle circumference $2 \pi R$, where $R$ is the circle radius $(\mathrm{B})$.

\section{Introduction}

Currently, everyone can learn to create artistic designs from books and the Internet. However, it is difficult for novices to adapt motifs created by other artists, because of skill gaps between novices and experts. Therefore, novices satisfy their willingness to create themselves by improving their skills by repeating practice. Also, adjustment of the level of difficulty in practice is indispensable to enhance the effect. Practice at an appropriate level of difficulty leads novices to flow state. The flow state through creative and other activities yields fulfillment and happiness [11]. Paper-cutting is an art performed by controlling a knife and cutting paper. The creation of paper-cutting helps with concentration and has a relaxing effect. Actually, creating paper-cutting has a distraction effect of temporarily suppressing negative thinking [7]. When the difficulty is inappropriate concerning the skill level, the flow state due to the creative activity decreases.

Our goal is to help novices get "optimal experience." Therefore in this paper, we aim to measure increases practice effect. The practice effect is affected by the difficulty level of the cutting pattern and the cutting skill of the user. We generalized the index of difficulty $(I D)$ based on the Steering law by patterning on straight lines and curves that compose the picture. One of the differences in cutting skill between novices and experts is the time to cut paper, so we compare the change of cutting time of each pattern.

In this paper, we developed a system to measure the cutting movement times $(M T)$ by creating an improved drawing display and stylus. From the two experiments, we verified that there was a difference in practice efficacy depending on the ID of the pattern to practice. We confirmed skill improvements by measuring changes in MT when novices repeatedly practiced with various $I D s$. Additionally, we mea- sured the reduction rate of $M T$ to investigate the effectiveness of practice with multiple IDs.

\section{Related Work}

The steering law quantifies the difficulty of a trajectory task with $I D$ and relates path steering time with the index in a linear fashion. The difficulty of steering through a straight tunnel is $I D_{s}=A / W$ (Fig. $\left.1-\mathrm{A}\right)$. And the difficulty of steering through a circular tunnel is $I D_{c}=2 \pi R / W$, where $A$ and $2 \pi R$ are the lengths of the tunnel and $W$ is the tunnel width (Fig. 1 -B) [1]. The Steering law can show as $M T=a$ $+b \times I D$ for both the straight and circular tunnels [1]. The $a$ and $b$ are empirically determined constants. We adapted the $I D$ and $M T$ in the Steering law to the difficulty level and creative time required for the paper-cutting pattern.

To date, many researchers have measured performance with various devices and for multiple shapes. For exam-

ple, researchers have investigated operations with multiple types of input device (mouse, stylus, touch panel, trackball) $[15,4,1]$. Moreover, several researchers have studied pen stroke gestures in letter writing [3] and the drawing of simple figures [9]. Curves have many deformations according to the curvature, and [10] researchers have investigated the Steering law that adapts to them. Additionally, shape-based differences have been investigated [16, 13]. [14] modeled the cutting behavior by scissors. Many researchers have examined intricate patterns regarding the Steering law.

We investigated that it is one pressure of difference between novices and experts by measuring the cutting action of paper-cutting. We developed a practice system that trains with a tracing movement about a pressure that is skilled [5], and a system that measures the cutting motion by attaching a blade to the tip of the stylus [6]. Moreover, other paper-cutting researchers have developed for lower 

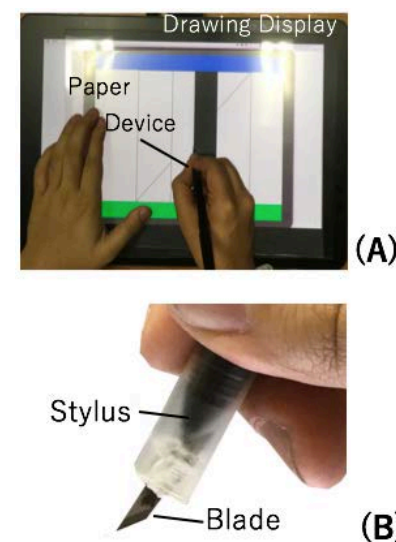

(B)

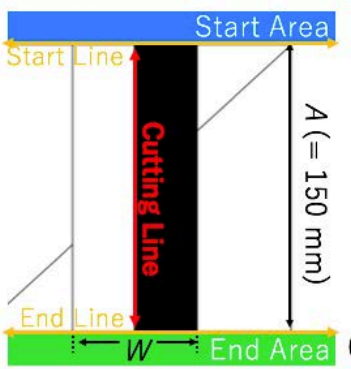

(C)

Figure 3: Participants cut paper placed on the display (A). A blade is attached to the tip of the stylus, and the gap is covered with resin (B). The display shows images of six types of straight lines and curves (C).

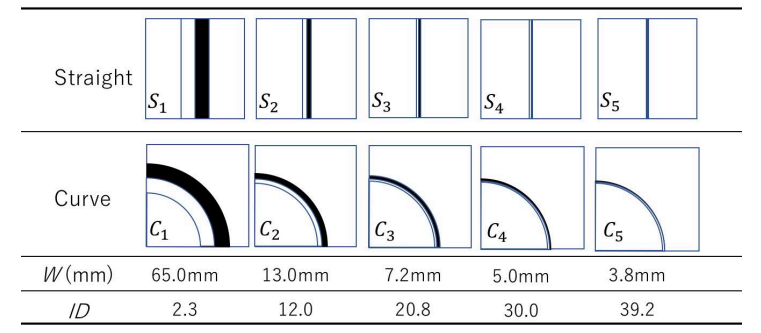

Figure 2: Straight-line patterns and curve patterns were composed of the same width and $I D$.

the fabricated barrier for novices, and promote the creating the paper-cut [8].

\section{Difficulty Level Measurements}

The artist cuts the border between a white and black area in a monochrome picture. We interviewed five instructors about the length of widths for novices. These instructors are experts, with five years of experience for three of the experts and six years of experience for two of the experts. From these interviews, we considered $13.0 \mathrm{~mm}$ to be the width that novices could cut smoothly $(S D=0.89)$, and the narrow width was $5.0 \mathrm{~mm}(\mathrm{SD}=0.31)$.

We calculated the $I D$ of these patterns using the Steering law. Based on our results, we designed ten images with various widths (Fig. 2). In these models, the ID monotonically increases and included widths that would be comfortable and difficult. All patterns were $150.0 \mathrm{~mm}$ in length.

\section{System for Measuring Skill Level}

Our system for measuring artist skill level consists of a drawing display and stylus (Fig. 3 -A). The display (Wacom Cintiq Pro16) shows the pictures $(3860 \times 2140$ pixel, $275 \mathrm{dpi}$ ) in descending order of $I D$. We modified this stylus to collect data by attaching the blade (NT BDC - 200 P) to the tip of the stylus (Wacom Pen Pro 2) (Fig. 3 -B). The display can obtain location and angle data for the grip pen via electromagnetic induction. Although the pen tip of the stylus and the surface of the screen do not touch, the display can recognize the stylus coordinates [12]. The user cuts the paper fixed to the tempered glass which covers to protect the display.

The user begins cutting the paper at the start location, moves along the cutting line, and ends at the end location (Fig. 3 -C). In our system, the timer starts when the knife passes the start line and stops when the blade crosses the end line. When the user cuts the paper beyond the width, the system beeps, signaling failure. In that case, the user cuts the same pattern again. Participants were instructed to perform the required operation as quickly and accurately as possible. We referred to the measurement method that many researchers have used for the series of processes and system instructions [2, 13].

\section{Experiment 1: Difference in MTs and Skill}

\section{Experiment Description}

The purpose of this experiment was to use MTs to assess changes in participant skill. The participants were 20 novices (average age: 27.4 years, SD: 1.18) who have never created paper-cutting. First, they cut five types of straight lines, next cut five types of curves. They repeated these actions ten times. After the experiment, we interviewed the participants on the difficulty level of the patterns.

\section{Result of MTs}

As a result, these graphs show the $M T$ that changed by repeating it up to 10 times (Fig. 4). From $S_{1}$ to $S_{3}$ and $C_{1}$ to $C_{2}, M T$ converged to about $5000 \mathrm{~ms}$. Additionally, MT in $S_{2}$ and $C_{2}$ decreased to a value close to that of $S_{1}$ and 


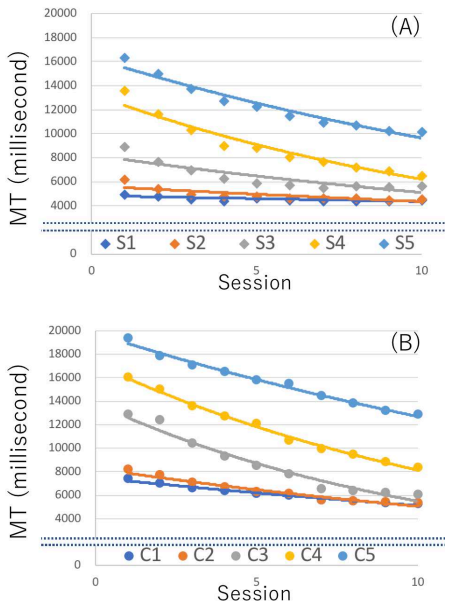

Figure 4: Horizontal axis represents cutting frequency, and vertical axis represents $M T$ in straight $(A)$ and Curve pattern (B).
$C_{1}$. From the interview, the participants felt that the width of $S_{2}$ was as comfortable as the width of $S_{1}$ (Fig. 4 -A). For the straight line, up until seven times, the amount of decrease from $S_{3}$ to $S_{4}$ is approximated. However, for $S_{5}$, less change was observed than for other patterns. The participants felt $S_{5}$ was particularly hard; therefore, it took more time than other patterns. The case involving curves showed similar results (Fig. 4 -B). Cutting along a curve requires rotating force, and is difficult compared to cutting along a straight line. Therefore, the amount of change in $C_{4}$ and $C_{5}$ decreased.

We confirmed that $M T$ changes according to $I D$. In particular, for low IDs, the decrease in MT converged by repeating cutting. Additionally, for patterns with high $I D$ s, the reduction in $M T$ is less pronounced than for low ID patterns. These causes are due to the difference between participant skill levels and design difficulty levels. From the above results, we measured the improvement of the skill from the change of $M T$.

\section{Experiment 2: Practicing with Various IDs}

\section{Experiment Description}

This purpose is to measure changes the skills to improve by practicing with the ID pattern corresponding to the own abilities. Participants are fifty inexperienced people (average age: 25.9 years, SD: 0.96) different from Experiment 1. They perform the following procedure: in Step 1, They cut each Figure 2 once. In Step 2, Each group practices with the various $I D$ in Figure 2. Moreover, in step 3, same as Step 1. We considered dividing the participants into 5 groups of 10 people based on the participants' average MT in Step 1. As a result, we confirmed that each groups skills have according to the close level. In Step 2, the participants practiced with one of Figure 2. The participants were divided into five groups as follows: Gr1 cut the $S_{1}$ and the $C_{1}$,
Gr2 cut the $S_{2}$ and the $C_{2}, \mathrm{Gr} 3$ cut the $S_{3}$ and the $C_{3}, \mathrm{Gr} 4$ cut the $S_{4}$ and the $C_{4}$, the last Gr5 cut the $S_{5}$ and the $C_{5}$. The groups performed two kinds of patterns five times.

\section{Result of Practice Efficacy}

Figure 5 shows the reduction rate of $M T$ between Step 1 and Step 3. As a result, the practice effect of the curve was weaker overall than the straight line, but the tendency of the impact was the like as the straight line. In Gr1 and Gr2, the reduction rate of all patterns remained a slight change (average: 0.91 ). The rate of decrease from Gr3 to Gr5 showed various changes depending on each ID. In particular, the results of $S_{3}, S_{4}$ and $C_{3}, C_{4}$ of $\mathrm{Gr} 3$ and $\mathrm{Gr} 4$ were smaller than the average 0.7 , which was a higher exercise effect than the other patterns. On the other hand, in Gr5, the reduction rate affects the exercise effect rather than $\mathrm{Gr} 1$ and Gr2, but it is not as effective as Gr3 or Gr4.

For these reasons, since $\mathrm{Gr} 1$ and $\mathrm{Gr} 2$ cut only patterns which are too simple in Step 2, the practice effect was low, and their skills could not be improved. Likewise, the $S_{5}$ and $C_{5}$ were the too difficult pattern to practice the practice effect. On the other hand, the result of Gr3 showed the highest exercise effect in the same pattern $\left(S_{3}\right.$ and $\left.C_{3}\right)$ practiced in Step 2. Similarly, the exercises in $S_{4}$ and $C_{4}$ were affected by effective improvement to Gr4s. $S_{3}, C_{3}$ and $S_{4}$, $C_{4}$ are patterns in which novices have reduced MTs in 10 iterative exercises, from the Experiment 1 . The difficulty level gives moderate stress to the participants and provides a high practicing effect. By repeatedly practicing those patterns, Gr3 and Gr4 were able to improve their skills more efficiently than the other participants.

\section{Discussion}

In this paper, we measured practice effect when user cut straight lines and curves. As a result of experiment 1, novices 


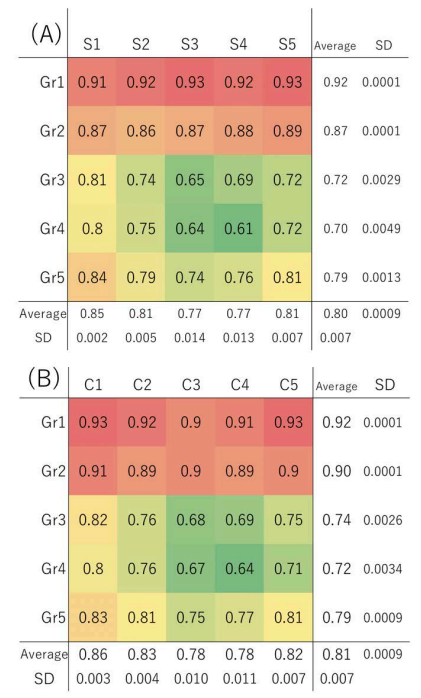

Figure 5: These heat maps show the reduced ratio of average time between Step1 and Step3 in the straight $(A)$ and curve pattern (B). can cut low ID patterns at a sufficient speed, so their MTs were constant in 10 times, and the effect is slight. Also, high IDs are a pattern which is too hard for practicing, and the amount of MTs decrease was small, and improvement of the skill of participants who performed with that pattern is also weak. On the other hand, the change of MTs in the pattern with moderate $I D$ showed a significant difference than those. Also in experiment 2, the result of them showed a more significant reduction rate than the other groups. From these results, we showed the effect of each ID on the change of cutting time measured by our system.

In these experiments, to improve the practice effect for novices, measurements were made by those who had no experience of cutting picture creation. Therefore, when targeting an intermediate person with experience of creation, the practice effect varies. Therefore, we measure the relationship between the skill level and the practice effect from the cutting action of the user. By doing this, we match the skill level according to users of various skills and give the user the best level of difficulty practice.

\section{Conclusion}

In this paper, we measured the improvement of participants skills from the change of it MT. We calculated the ID of the line based on the steering law. Our system can measure the $M T$ from various ID patterns cut by users. We investigated the practice effect by $I D$ from two experiments. From these results, we confirmed the practice effect of each difficulty level on the change of cutting time.

In future research, we will create a skill map to generalize the cutting skill. The skill map will be able to measures the difficulty level optimal for the skill level of the user, and the novices will be able to practice with optimal difficulty according to their changing his/her skills.

\section{REFERENCES}

1. Johnny Accot and Shumin Zhai. 1999. Performance Evaluation of Input Devices in Trajectory-based Tasks: An Application of the Steering Law. In Proceedings of the SIGCHI Conference on Human Factors in Computing Systems (CHI '99). ACM, New York, NY, USA, 466-472. DOI :

http://dx.doi.org/10.1145/302979.303133

2. Johnny Accot and Shumin Zhai. 2001. Scale Effects in Steering Law Tasks. In Proceedings of the SIGCHI Conference on Human Factors in Computing Systems (CHI '01). ACM, New York, NY, USA, 1-8. DOI : http://dx.doi.org/10.1145/365024.365027

3. Xiang Cao and Shumin Zhai. 2007. Modeling Human Performance of Pen Stroke Gestures. In Proceedings of the SIGCHI Conference on Human Factors in Computing Systems (CHI '07). ACM, New York, NY, USA, 1495-1504. DOI :

http://dx.doi.org/10.1145/1240624.1240850

4. Oryx Cohen, Shawna Meyer, and Erik Nilsen. 1993. Studying the Movement of High-tech Rodentia:

Pointing and Dragging. In INTERACT '93 and CHI '93 Conference Companion on Human Factors in

Computing Systems (CHI '93). ACM, New York, NY, USA, 135-136. DOI :

http://dx.doi.org/10.1145/259964.260149

5. Takafumi Higashi and Hideaki Kanai. 2016. Instruction for Paper-cutting: A System for Learning Experts' Skills. In Proceedings of the 2016 ACM on Interactive Surfaces and Spaces (ISS '16). ACM, New York, NY, USA, 457-460. DOI :

http://dx.doi.org/10.1145/2992154.2996872

6. Takafumi Higashi and Hideaki Kanai. 2017. Stylus Knife for Paper-cutting: A System for Controlling a 
Knife. In Proceedings of the 2017 CHI Conference Extended Abstracts on Human Factors in Computing Systems (CHI EA '17). ACM, New York, NY, USA, 2638-2645. DOI :

http://dx.doi.org/10.1145/3027063.3053224

7. Haruyuki Ishikawa and Fusako Koshikawa. 2018. The effect of distraction with recalling objects of rumination on mood, and the evaluation of ruminative thoughts. Journal of Health Psychology Research 30, 2 (2018), 65-73. DOI :

http://dx.doi.org/10.11560/jhpr.160816047

8. Lijuan Liu, Yang Chen, Pinhao Wang, Yizhou Liu, Caowei Zhang, Xuan Li, Cheng Yao, and Fangtian Ying. 2018. Papercut: Digital Fabrication and Design for Paper Cutting. In Extended Abstracts of the 2018 $\mathrm{CHI}$ Conference on Human Factors in Computing Systems (CHI EA '18). ACM, New York, NY, USA, Article LBW078, 6 pages. DOI :

http://dx.doi.org/10.1145/3170427.3188522

9. A. Chris Long, Jr., James A. Landay, Lawrence A. Rowe, and Joseph Michiels. 2000. Visual Similarity of Pen Gestures. In Proceedings of the SIGCHI Conference on Human Factors in Computing Systems (CHI '00). ACM, New York, NY, USA, 360-367. DOI : http://dx.doi.org/10.1145/332040.332458

10. Mathieu Nancel and Edward Lank. 2017. Modeling User Performance on Curved Constrained Paths. In Proceedings of the $2017 \mathrm{CHI}$ Conference on Human Factors in Computing Systems (CHI '17). ACM, New York, NY, USA, 244-254. DOI : http://dx.doi.org/10.1145/3025453.3025951

11. Martin E. P. Seligman. 2012, Flourish: A Visionary New Understanding of Happiness and Well-being (2012).
12. Masato Soga, Shota Kuriyama, and Hirokazu Taki. 2009. Transactions on Edutainment III. Springer-Verlag, Berlin, Heidelberg, Chapter Sketch Learning Environment with Diagnosis and Drawing Guidance from Rough Form to Detailed Contour Form, 129-140. http:

//dl.acm.org/citation. cfm?id=2172394.2172407

13. Shota Yamanaka and Homei Miyashita. 2016. Modeling the Steering Time Difference Between Narrowing and Widening Tunnels. In Proceedings of the $2016 \mathrm{CHI}$ Conference on Human Factors in Computing Systems (CHI '16). ACM, New York, NY, USA, 1846-1856. DOI : http://dx.doi.org/10.1145/2858036.2858037

14. Shota Yamanaka and Homei Miyashita. 2018 Paper-cutting operations using scissors in Drury's law tasks. Applied Ergonomics 69 (2018), 32 - 39. DOI : http://dx.doi.org/https: //doi.org/10.1016/j.apergo.2017.12.018

15. Stanislaw Zabramski. 2011. Careless Touch: A Comparative Evaluation of Mouse, Pen, and Touch Input in Shape Tracing Task. In Proceedings of the 23rd Australian Computer-Human Interaction Conference (OzCHI '11). ACM, New York, NY, USA, 329-332. DOI : http://dx.doi.org/10.1145/2071536.2071588

16. Stanislaw Zabramski, Suman Shrestha, and Wolfgang Stuerzlinger. 2013. Easy vs. Tricky: The Shape Effect in Tracing, Selecting, and Steering With Mouse, Stylus, and Touch. In Proceedings of International Conference on Making Sense of Converging Media

(AcademicMindTrek '13). ACM, New York, NY, USA Article 99, 5 pages. DOI :

http://dx.doi.org/10.1145/2523429.2523444 